\title{
Light Absorption near Threshold with Phonon Participation for Impurities in Semiconductors
}

\author{
M. A. Amato \\ Instituto de Física, Universidade de Brasília \\ 70910-900, Brasília, DF, Brazil
}

Recebido em 7 de junho, 2002. Aceito em 3 de setembro, 2002.

\begin{abstract}
It is presented a simple model for the calculation of the transition rate for impurities in semiconductors in which electron-phonon interaction is taken into account in a second order time dependent perturbation theory. This result shows the explicit dependence of the transition rate on the phonon density of states and that the absorption curve of a semiconductor is modulated by the phonon structure.
\end{abstract}

In recent years progress has been made in investigating the physical properties of impurity centres in semiconductors either theoretically or experimentally. For deep level impurities their identification and characterisation remains as a difficult problem it is still an important and active field in semiconductor research [1]. On the other hand, many of the shallow impurities are by now well understood. The effective mass theory of shallow impurities [2] led to the well-known hydrogenic theory providing both conceptual guidance and, in many cases, quantitative predictions about the binding energies [3].

Deep impurities are so different from shallow impurities that extension of effective mass theory has not proved to be successful in understanding their properties. Their distinguishing feature is in the characteristic of the long range potential. Shallow impurities are controlled by long range potentials, whereas the presence of short range potential produces only a slight chemical shift. For deep impurities this relative importance of the potential works on the opposite way.

Some theoretical approaches are used to tackle the impurity problem. There are first principles type of approaches that aim at a truly quantitative assessment of carefully selected impurity-host systems [4][5]. They employ self-consistent pseudopotential techniques, which provides a very detailed information about electronic and vibronic properties of specific defects, such as charge densities [6], lattice relaxation around defects [7], and energy levels. The semiempirical approaches offer an imprecise but global view of deep impurities in many different hosts [8] which employ a modern version of tight binding theory [9], augmented by the Koster-Slater Green's function technique. These approaches focus mainly on chemical trends in the im- purity levels energies rather than to give quantitative agreement with existing data.

What makes difficult to obtain an accurate solution to the problem is the presence of the interaction between the electrons and the lattice. The model generally used for a quantitative treatment of electronphonon interaction is the so called configuration coordinate model. Its formalism has developed a long time ago and reviews exist on this topic for semiclassical as well as quantum treatment [10][11][12][13]. In its original form this model is worked out for transitions within localised states, and it has been applied successfully to such systems as F-centres in alkali halides and rare-earth impurities in semiconductors. It is also widely accepted for studying transitions related to deep centres, mainly due to a bigger Franck-Condon shift. However, for a small Franck-Condon shift, which occur for some deep centres, it would be appropriate to model transitions through indirect processes. This would also allow to study transitions connected to phonon sidebands. Indirect processes can play a significant role in determining the shape and the position of the peaks in the phonon side band structure of deep impurities in semiconductors [14].

It is generally accepted that even were a full theory available, it would be necessary to extract from it an acceptable, simplified model which could be used to define the principal measurable quantities and to provide a useful terminology for describing experimental results. The presence of such a model is of some importance and we attempt in this article to illustrate what experimental features lend themselves most readily to modelling.

Whilst many of these subjects can readily be discussed in a qualitative way in an undergraduate class, 
it is difficult to go into the theory underlying the absorption process unless the students have a sound background in time dependent perturbation theory. However, some important quantitative features of the process can be conveyed by elementary methods described in this paper.

In this paper we would like to enlighten the relevance of the electron-phonon interaction in the optical absorption spectra due to the presence of impurities in semiconductors. As we have pointed the configuration coordinate model is a generally accepted one to provide the amount of interaction with phonons. However, on a different basis we propose a more simple treatment, which extract from the experimental results the characteristic phonon frequency that assists the electronic transition.

For sake of convenience we restrict our discussion to the case of donor impurity, although this could also be formulated in terms of acceptors. We shall start with the Hamiltonian

$$
H=H^{c r y s}+H^{i n t}
$$

where the perturbation $H^{i n t}$ is given by

$$
H^{i n t}=H^{e-p}+H^{r a d}
$$

The $H^{\text {rad }}$ contains the usual description in terms of electronic and phonon band states, and $H^{e-p}$ takes into account the electron-photon interaction only. For the electron-phonon interaction one takes [9]

$$
H^{e-p}=\sum_{\vec{k}} \sum_{\vec{q}} V(\vec{q}) a_{\vec{q}+\vec{k}}^{\dagger} a \vec{k}^{\left(b \vec{q}+b_{-} \vec{q}\right)}
$$

with being $a \vec{k}(b \vec{q})$ the electron (phonon) operators.

Making use of the Fermi Golden Rule, the transition rate is given by

$$
W=\frac{2 \pi}{\hbar} \sum\left|\left\langle f\left|H_{f}^{i n t}\right| I\right\rangle\right|^{2} \delta\left(E_{f}-E_{I}\right)
$$

where $\langle f|$ and $\langle I|$ are the conduction band and trap states, respectively. The wave function of the electron in the presence of the impurity potential may be expanded in terms of Bloch functions in the form

$$
\Psi_{I}=\frac{1}{\sqrt{V}} \sum_{n, \vec{k}} A_{n, \vec{k}}\left|\phi_{n, \vec{k}}\right\rangle
$$

This form applies to both trap and conduction band state, though the coefficients will be generally different in the two cases. Proceeding further and considering only phonon emission, for a donor impurity one gets for the transition rate in the long wavelength approximation[19]

$$
\begin{aligned}
W \approx & \frac{2 \pi}{\hbar V} \sum_{n, n \prime, \alpha} \sum_{k, \vec{k}, \vec{q}} A_{n, \vec{k}} A_{n^{\prime}, \overrightarrow{k^{\prime}}}^{*} \times \\
& \times \sum_{i}\left|\frac{\left\langle\phi_{n, \vec{k}}\left|H^{r a d}\right| \phi_{i, \vec{k}}\right\rangle\left\langle\phi_{i, \vec{k}}\left|H^{e-p}\right| \phi_{n^{\prime}, \overrightarrow{k^{\prime}}}\right\rangle}{E_{\vec{k}}-E_{i}}\right| \times \delta\left(E_{\overrightarrow{k^{\prime}}}-E_{I}-\hbar \omega+\hbar \Omega_{\vec{q}}\right)
\end{aligned}
$$

$\hbar \Omega \frac{\alpha}{q}$ is the $\alpha$ th branch phonon energy with wave vector $\vec{q}$ and the index $i$ refers to intermediate states. In an indirect process momentum conservation requires $\overrightarrow{k^{\prime}}-\vec{k}=\vec{q}$, and for $n=n^{\prime}$ equation (6) can be further simplified. The coefficients in the wave function expansion can be calculated according to the impurity model [15]

Equation (6) may be seen as composed of two major terms. The first describing the structure of the transition rate, and the second incorporating the density of states. In fact, following the prescription of [16] this equation can be factorised into a term containing the matrix element and a sum over the delta functions leading to the simple product structure. The transition rate can be expressed as a product of the squared matrix elements and the density of final states

$$
W \approx|M|^{2} \sum_{\vec{q}, \alpha} \delta\left(E_{\overrightarrow{k^{\prime}}}-E_{I}-\hbar \omega+\hbar \Omega \frac{\alpha}{q}\right)
$$

with 


$$
\begin{aligned}
|M|^{2} & =\frac{2 \pi}{\hbar V} \sum_{n, n \prime} \sum_{k}, \vec{k} \\
& \times \sum_{n, \vec{k}} A_{n^{\prime}, \overrightarrow{k^{\prime}}}^{*}\left|\frac{\left\langle\phi_{n, \vec{k}}\left|H^{\text {rad }}\right| \phi_{i, \vec{k}}\right\rangle\left\langle\phi_{i, \vec{k}}\left|H^{e-p}\right| \phi_{n^{\prime}, \overrightarrow{k^{\prime}}}\right\rangle}{E_{\vec{k}}-E_{i}}\right|^{2}
\end{aligned}
$$

We may argue that this approximation is justified by saying that the trap state is very localised implying that such a state has Fourier components along all the different $k^{\prime} s$ in the Brillouin zone. It is obvious that such analysis fails whenever the matrix element vanishes due to symmetry of the involved states. In summary, this expresses the transition rate as a product of the squared matrix elements and the density of final states.

Equation (8) shows in a simple way how the phonon density of states modulates the transition rate. The absorption threshold in the presence of phonons is shifted by the amount of phonon energy supplied toward lower energies for phonon absorption or toward higher energies for phonon emission [17][18]. Any further calculation should take into account the effects of band structure. As an exercise, for a parabolic band structure, and a single phonon frequency, one can easily reproduces the power law for the absorption coefficient, $\alpha(\hbar \omega)[20]$.

In referring to the validity of the present model, may be pointed out that it takes into account only single phonon processes, or it is a first order electron-phonon model. In the case of deep impurities, the electron relaxation is assisted by many phonons, so that it is analysed by higher order perturbation theory, and the present analysis fails, although for small lattice distortion, the model can be applied to the understanding of the behaviour of a deep impurity near the optical threshold. Finally, the above result does not require fine details of the electron-phonon interaction, but unfortunately it does not apply to regions well above threshold.

In order to avoid misunderstanding in the subject due to the simplicity of the above model a word about the wavefunctions related to the impurities and conduction band states ought to be said, so that a proper calculation of the matrix elements can be performed. In fact the determination of the wavefunctions is a rather difficult problem due to the potential introduced by the foreign atom in the host system. The translational symmetry is lost and the wavefunctions are not simple the
Bloch function. For bound states, to circunvent the problem in the solution of the Schroedinger wave equation one has to model either the wavefunction or the potential. For the later, the simplest of these models is the hydrogenic model, for shallow impurities and the Lucovsky model for deep impurities. In the conduction band the electronic wave function is more complicated by scattering of electrons by the potential. The simplest solution has been to adopt as the wave function that for a plane wave. This choice is obviously a good one for a neutral centre which scatter only weakly and for spread out localized states at high free energies, but by no means an obvious choice for charged states

In summary, the model described in this paper is undoubtedly over-simplistic, and should not be regarded as anything more than a conceptual aid. On the other side, it does provide an easily understood introduction to the subject, and may enable some useful quantitative results to be obtained without a detailed quantum mechanical calculations.

\section{References}

[1] S. Pantelides, Perspectives in the Past, Present, and Future of Deep Centers, pp. 1-85, in Deep Centers in Semiconductors: A State of the Art Approach, S. Pantelides (ed.), 1997, Gordon and Breach.

[2] J. M. Luttinger and W. Kohn. Phys. Rev. 97, 969 (1955).

[3] W. Kohn, Shallow Impurity States in Silicon and Germanium, in Solid State Physics, F. Seitz and D. Turnbull (eds.), 5, 257-320 (1957).

[4] G. A. Baraff, and M. Schluter, Phys. Rev. B19, 4965 (1979).

[5] M. Jaros, Adv. Phys. 29, 409 (1980).

[6] M. Jaros, C. D. Rodriguez, and S. Brand, Phys. Rev. B19, 3137 (1979).

[7] G. A. Baraff, E. D. Kane, and M. Schluter, Phys. Rev. B21, 4965 (1980). 
[8] J. Bernholc, S. Pantelides, N. D. Lipari, and A. Balderechi, Solid State Commun. 37, 705 (1981).

[9] H. P. Hjalmarson, P. Vogl, and J. D. Dow, Phys. Rer. Lett. 44, 810 (1980).

[10] P. Vogl, H. P. Hjalmarson, and J. D. Dow, J. Phys. Chem. Solids 44, 365 (1983).

[11] K. Huang, and A. Rhys, Proc. Royal Soc. A204, 406 (1950).

[12] M. Lax, J. Chem. Phys. 20, 1752 (1952).

[13] J. J. Markham, Rer. Mod. Phys. 31, 956 (1959).

[14] H. Dai, M. A. Gundersen, C. W. Myles, and P. G. Snyder, Phys. Rev. B37, 1205 (1988).

[15] M. A. Amato, and B. K. Ridley, J. Phys. C: Solid State Phys. 13, 2027 (1980).
[16] H. N. Nazareno, and M. A. Amato, J. Phys. C: Solid State Phys. 15, 2165 (1982).

[17] M. A. Amato, M.C. Arikan, and B. K. Ridley, in Proc. on III-IV Semi-insulating Materials Conference, pp.249-252, G. J. Rees (ed.), 1980, Shiva Publishing Ltd.

[18] B. Monemar, and L. Samuelson, Phys. Rev. B18, 809 (1978); Phys. Rev. B18, 830 (1978).

[19] In the long wavelenght approximation the photon wavevector is negligible if compared to the electron wavevector in magnitude.

[20] The transition rate and the absorption coefficient are related through the equation $\alpha(w)=\frac{W V}{\langle N\rangle c} \eta$ with $\eta$ being the refractive index of the medium, and $c$ the velocity of light in vacuum. 